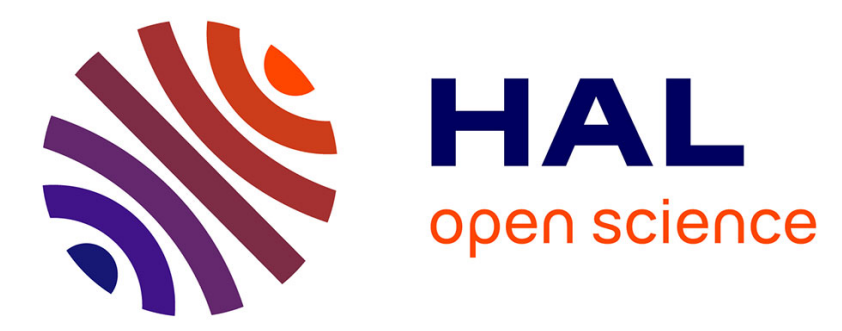

\title{
Vers l'identification de paniers de biens et de services liée à la demande locale dans les territoires d'élevage: illustration à partir de la Planèze de Saint Flour et du bocage bourbonnais
}

Dominique Vollet, S. Said

\section{To cite this version:}

Dominique Vollet, S. Said. Vers l'identification de paniers de biens et de services liée à la demande locale dans les territoires d'élevage: illustration à partir de la Planèze de Saint Flour et du bocage bourbonnais. Géocarrefour - Revue de géographie de Lyon, 2018, 92 (3), 10.4000/geocarrefour.11155 . hal-02608876

\section{HAL Id: hal-02608876 \\ https: / hal.inrae.fr/hal-02608876}

Submitted on 16 May 2020

HAL is a multi-disciplinary open access archive for the deposit and dissemination of scientific research documents, whether they are published or not. The documents may come from teaching and research institutions in France or abroad, or from public or private research centers.
L'archive ouverte pluridisciplinaire HAL, est destinée au dépôt et à la diffusion de documents scientifiques de niveau recherche, publiés ou non, émanant des établissements d'enseignement et de recherche français ou étrangers, des laboratoires publics ou privés. 


\title{
Vers l'identification de paniers de biens et de services liée à la demande locale dans les territoires d'élevage : illustration à partir de la Planèze de Saint Flour et du bocage bourbonnais
}

\section{Résumé}

A partir d'enquêtes qualitatives et quantitatives menées auprès des consommateurs et des filières de production agricole de deux petites zones auvergnates caractérisées par la diversité de leur système d'élevage (le Bocage Bourbonnais dans l'Allier et la Planèze de Saint-Flour dans le Cantal), cet article présente l'originalité d'appliquer le modèle de biens complexes territorialisés (ou paniers de biens et de services) à la consommation locale. Plusieurs spécificités fortes sont mises en évidence : un panier moyen constitué d'un mélange de bœuf, de porc et de légumes, une capacité de consommateurs à apprécier la typicité des produits, un ensemble de services davantage lié à la vitalité territoriale qu'aux aménités paysagères des systèmes d'élevage.

\begin{abstract}
Based on qualitative and quantitative surveys of consumers and agricultural production sectors of two small Auvergne regions characterized by the diversity of their farming system (the "Bocage Bourbonnais" in the Allier and the "Planèze of Saint-Flour" in the Cantal), this article presents the originality of applying the model of territorialized complex goods (or baskets of goods and services) to local consumption. Several strong specificities are highlighted: an average basket consisting of a mixture of beef, pork and vegetables, a capacity of consumers to appreciate the specificity of the products, a set of services more related to territorial vitality than to the landscape amenities of farming systems.
\end{abstract}

\section{Introduction}

A l'heure de la mondialisation et de la standardisation, l'agriculture doit faire face à un enjeu considérable, nourrir une population grandissante dans des délais de plus en plus courts dans un contexte de changement global. Les attentes sociétales, notamment celles des consommateurs, ne cessent d'évoluer. Ces derniers ont tendance à se préoccuper davantage de l'origine, du mode de production et de la qualité des produits, mais ils accordent aussi de l'importance à la préservation de l'environnement. Ainsi, de nouvelles politiques de différenciation voient le jour en renforçant le lien agriculture-environnement ou en développant une nouvelle stratégie commerciale fondée sur l'origine et/ou la qualité (Pecqueur, 2001 ; Hirczak, 2011). Pour pallier cette uniformisation des produits agricoles, certains décident de se distinguer grâce à des signes officiels de qualité valorisant la production et les aménités environnementales qui constituent un atout favorable et attractif pour le développement des territoires (Roux et al., 2007).

Les produits locaux se trouvent au cœur de ces stratégies de différenciation, particulièrement ceux qui relèvent des biens complexes territorialisés ${ }^{1}$. Ce type de stratégie a été mis en

\footnotetext{
${ }^{1}$ D'après Hirczak et al. 2008, Il s'agit de différents biens et services reliés par une ou plusieurs caractéristiques communes qui leurs confèrent une «identité partagée » (même territoire, même origine, produits complémentaires)
} 
évidence dans le cas de valorisation touristique associant soit des produits végétaux (huile, vins par exemple) soit des produits animaux faciles à emporter (fromages notamment) (Roux et al., 2007 ; Hirzak et al., 2004). Ce travail porte sur les produits locaux issus de l'élevage (viande y compris) avec l'originalité d'analyser les possibilités et les conditions de mise en œuvre de stratégies de différenciation pour une consommation non pas touristique mais s'adressant à des consommateurs locaux. L'objectif est d'étudier les conditions de valorisation de ces produits notamment par l'existence d'une demande pour des produits complémentaires ou d'appel (maraichage par exemple). Pour répondre à ces questions, deux études de cas, partageant comme point commun l'existence de canaux locaux de valorisation de leurs produits de qualité, ont été choisies (le Bocage Bourbonnais et la Planèze de SaintFlour). Leurs différences fortes en termes de types d'élevage et de structuration des filières permettront de tirer des enseignements plus génériques.

Une première partie explicitera le cadre théorique retenu (modèle de biens complexes territorialisés). La seconde partie sera consacrée à rendre compte de la démarche retenue et des critères de choix des deux terrains d'application. Une troisième partie détaillera les résultats qui tendent à montrer l'existence d'un panier de biens complexes territorialisés très spécifiques aux consommateurs locaux, que ce soit dans sa composition en biens marchands ou en services joints.

\section{Une application originale du modèle des biens complexes territorialisés à la consommation locale}

Pour apprécier les possibilités de valorisation des produits issus de l'élevage par la consommation locale, nous avons retenu le modèle des biens et services complexes territorialisés. Lorsque le modèle concerne un seul bien ou service, la valorisation de ce dernier peut être basée sur la satisfaction du consommateur qui ne provient pas du bien acheté mais de l'ensemble des attributs qui le caractérise (Lancaster, 1991). En revanche, lorsque le modèle porte sur plusieurs biens ou services partageant la même identité, l'application du principe de Lancaster introduit l'idée de croiser leur caractéristiques communes et distinctes en leur donnant la possibilité d'être associées (Mollard et al., 2005). C'est ce qu'on appelle des biens complexes, tels que les produits touristiques de même origine. Par ailleurs, lorsque ces biens complexes sont ancrés au territoire de production par les caractéristiques qui les définissent, on parle alors de biens complexes territorialisés. La viande et le fromage constituent de véritables biens complexes territorialisés dans la mesure où le consommateur les achète pour leur caractéristiques communes telles que les qualités sanitaire et organoleptique mais également telles que l'image de marque (plus ou moins ancrée dans le territoire de production) et l'existence de services environnementaux (paysage, biodiversité) qui peuvent être générés conjointement avec leur production marchande.

Ces produits différenciés par l'origine et/ou la qualité de production sont à l'origine d'une « rente ». Le potentiel de rente différentielle d'un produit donné sur un territoire peut être estimé en réalisant une analyse statistique comparée de séries de prix homogènes concernant le produit ou service observé et son substitut générique (Pecqueur, 2001). Les séries statistiques utilisées doivent être assez longues afin de vérifier le caractère à la fois durable et significatif des écarts de prix par rapport à un produit de référence substituable. Par ailleurs, le potentiel de rente différentielle dépend également du prix que le consommateur est prêt à payer pour acquérir ce bien : il s'agit du consentement à payer (CAP). Ce dernier peut être affecté par plusieurs facteurs tels que la rareté du produit, l'image qu'il véhicule, le degré de substituabilité avec d'autres biens, et l'intensité de la demande. Toutefois, dans le cas des formes de distribution analysées dans la littérature, particulièrement les circuits courts, le 
CAP est également affecté par divers types de «proximités ». Bergadaà et Del Bucchia (2009) ont identifié entre un client et une enseigne de distribution alimentaire cinq dimensions de la proximité : les proximités d'accès, identitaire (proximité avec les valeurs mises en avant), relationnelle (relations directes consommateurs-producteurs-vendeurs), de processus (importance accordée au fonctionnement interne du point de vente) et fonctionnelle (exemple : avoir un choix large de produits). Héraut-Fournier et al. (2012) ont montré le rôle important joué par les proximités identitaire, relationnelle et de processus dans le cas des circuits courts agro-alimentaires. Au total, le survey réalisé par Feldman et Hamm (2015) a montré que les produits locaux ne sont généralement pas perçus comme chers par les consommateurs (contrairement aux produits biologiques).

Du point de vue du producteur, plus le CAP des consommateurs est élevé et plus il constitue une valeur ajoutée aux produits différenciés se traduisant par un revenu spécifique éventuel et l'existence d'une rente de qualité territoriale (RQT) potentielle (Mollard, 2001 ; Hirczak et Mollard, 2004). La RQT potentielle ou effective est issue de la combinaison de deux rentes :

- La rente de qualité existe pour des biens ayant des propriétés supérieures qui les différencient des biens industriels de qualité générique qualifiés par une rente nulle.

- La rente territoriale existe pour des biens ancrés au territoire en fonction de la productivité et de la rareté des ressources de leur origine d'implantation.

Si l'existence d'une rente de qualité territoriale pour des biens et services donnés est avérée, cela signifie que toutes ou partie des aménités territoriales (paysage, biodiversité animale ou végétale) produites conjointement avec la production agricole sont valorisées dans le prix de ces produits. Les consommateurs sont prêts à payer plus cher ce type de produit dans la mesure où ils apprécient la qualité des aménités paysagères. La pérennité de la RQT est à la fois fonction de la coordination des stratégies publiques et privées d'offre et de leur adéquation avec les attentes et les préférences du consommateur. Ces deux conditions favorisent plus ou moins "l'effet panier", défini par des produits issus d'un même territoire et composés de biens et services complémentaires ayant une valorisation mutuelle. L'existence d'un effet panier de biens et de services a été mise en évidence dans un premier cas de figure dans les Baronnies : l'AOP huile de Nyons est associée à d'autres produits de terroir qui se valorisent mutuellement et qui diffusent la même image du territoire (Mollard, 2001 ; Moalla, 2005). Dans l'Aubrac, il s'agit plutôt d'un panier comprenant le fromage AOP Laguiole, le couteau et l'aligot, qui a émergé dans les années 2000 (Angeon et Vollet, 2010) et a été conforté par le projet développé par le Parc Naturel Régional de l'Aubrac créé en mai 2018. Dans les deux cas, la présence simultanée d'un ensemble composé d'aménités naturelles, du patrimoine bâti, de plusieurs produits de qualité et du tourisme (qui agit comme une véritable «clé d'entrée ») confère à l'ensemble des biens et services produits une valeur ajoutée supplémentaire génératrice d'un processus de développement local.

Les territoires analysés jusqu'alors par le modèle des biens complexes territorialisés sont essentiellement des zones plutôt touristiques où la consommation simultanée de biens et de services est le fait (du moins dans un premier temps) d'une consommation extérieure au territoire (Mollard et al., 2005). De plus, les produits concernés ont pour la plupart des caractéristiques de conservation et de praticité permettant une consommation touristique effectuée sur place ou à distance tels que le fromage, l'huile, l'aligot ou le couteau. Notre travail complète cette littérature en se focalisant principalement sur la viande, un bien moins emblématique et plus ordinaire dans la consommation des ménages. Cependant, bien que sa consommation (toutes viandes confondues sauf le poulet) enregistre un recul depuis trois ans dans un contexte de prix moyen à la hausse (Ministère de l'Agriculture et de l'Alimentation, 
2018), la France possède le plus gros cheptel bovin d'Europe (Institut de l'élevage, 2017) en utilisant $41 \%$ de sa surface Agricole Utile (SAU) en prairie. Puis pour la viande, la dimension territoriale est moins facile à percevoir par le consommateur pour certains signes de qualité, notamment le Label Rouge, qui est avant tout lié à une race. Par exemple, une viande Label Rouge répond à des exigences définies dans un cahier des charges qui porte d'abord sur la race, également les conditions de production, l'élaboration et la qualité du produit mais qui ne tient pas compte de l'ancrage territorial de ce dernier. Ainsi, la viande comme composante d'un modèle de biens complexes territorialisés représente-t-elle un cas plus complexe à étudier en utilisant une approche qui se base sur la consommation locale et non touristique.

\section{Le choix d'une méthode adaptée à deux études de cas très représentatives des territoires d'élevage}

Avec $70 \%$ de sa SAU en herbe, la Région Auvergne Rhône-Alpes est la première prairie de France. Deux terrains d'étude ont été retenus dans cette région en raison de leur contribution significative dans les activités de l'élevage, leur dimension faiblement touristique et l'existence d'une consommation locale significative. Notre choix s'est arrêté à deux territoires ruraux ayant des caractéristiques contrastées. Le premier, Bourbon l'Archambault dans le Bocage Bourbonnais dans l'Allier, est considéré comme un bassin allaitant avec une production agricole marquée par une forte compétition entre l'élevage et les cultures. Le second, la Planèze de Saint Flour/Margeride dans le Cantal, est caractérisé par une importante activité d'élevage laitier et une politique favorisant la valorisation de l'herbe (Tableau 1).

Par ailleurs dans les deux cas, les intercommunalités sont fortement impliquées dans le soutien des activités favorisant le développement de l'élevage. C'est le cas des programmes Leader dans les deux territoires : Le Groupe d'Action Locale (GAL) «Territoire Bourbon Pays de Moulin Auvergne, terre de ressources, sources d'attractivité » (2014-2020) a dédié l'une de ses fiches-actions à «valoriser les productions et les produits locaux » (qui représente environ $10 \%$ de l'enveloppe). Le GAL «Pays de Saint-Flour : construisons ensemble un territoire ouvert et solidaire » est plus en retrait de ce point de vue.

De point de vue géographique (Figure 1), les deux territoires sont situés dans des contextes très différents : le Bocage Bourbonnais est caractérisé par un terrain de plaine ondulé situé autour de $200 \mathrm{~m}$ d'altitude avec un paysage composé d'un maillage de parcelles entourées de haies, enserrant des zones destinées à l'élevage avec la présence de chênes et de forêts. En revanche, la planèze de Saint-Flour est un vaste plateau volcanique entre 700 et $1000 \mathrm{~m}$ d'altitude faisant partie des Monts du Cantal.
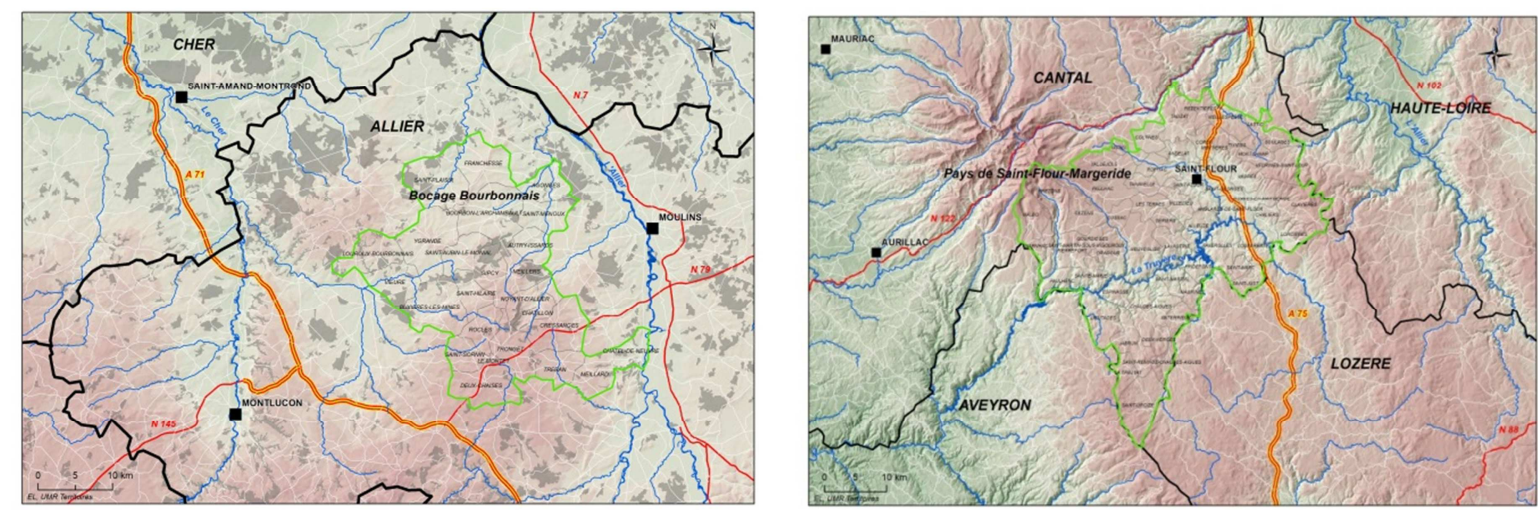
Figure 1 : Localisation des communautés de communes du Bocage Bourbonnais et de la Planèze de Saint-Flour (Source : UMR Territoires, Réalisation : Eric Langlois)

Dans les deux cas, l'agriculture représente un peu moins de $20 \%$ de l'emploi total, cinq fois plus que la moyenne nationale (Tableau 1). Inversement, l'industrie et le secteur tertiaire sont bien moins présents qu'au niveau national et même du massif central (environ $10 \%$ de différence pour l'emploi tertiaire). La population totale baisse sur longue période depuis l'après-guerre et s'est poursuivie depuis les années 70 alors qu'elle a progressé pour l'ensemble du massif et pour l'ensemble de la France. Le taux de chômage des 15-64 ans est supérieur à la moyenne du massif central pour le Bocage Bourbonnais alors qu'il est inférieur dans la Planèze de Saint-Flour. Ce sont deux territoires ruraux, en recherche de développement de produits spécifiques pour résister à la compétitivité et à la concurrence des régions productrices en produits standards (Tableau 2). Les stratégies d'acteurs qui portent la valorisation des produits agricoles sont cependant très différentes : En ce qui concerne le Bocage Bourbonnais, les coordinations privées comme publiques se sont révélées plutôt efficaces pour valoriser les produits de qualité. Il s'agit notamment de la $\mathrm{Sicaba}^{2}$, qui valorise sur place et à distance l'ensemble des viandes locales. La Communauté de communes porte également cette thématique avec une attention pour l'agriculture biologique (pour la viande comme le maraichage). Dans la zone de Saint-Flour, la communauté de communes est également attentive à la valorisation des produits locaux mais les initiatives restent limitées d'un point de vue quantitatif (lentilles, production de laitage frais) tandis que les 3 fromages AOP produits sur la zone sont essentiellement le fait de grands groupes agro-alimentaires (à l'exception d'une coopérative fromagère qui a misé sur un créneau spécifique de Cantal).

Tableau 1 : Chiffres clés des communautés de communes du Bocage Bourbonnais et de la Planèze de Saint-Flour/Margeride (Source : INSEE) en 2014

\begin{tabular}{|l|c|c|c|}
\hline & Bocage Bourbonnais & $\begin{array}{c}\text { Pays de Saint- } \\
\text { Flour/Margeride }\end{array}$ & Massif Central \\
\hline Nombre de communes & 25 & 29 & $/$ \\
\hline Siège & Bourbon l'Archambault & Saint-Flour & $/$ \\
\hline Superficie & $403 \mathrm{~km}^{2}$ & $622 \mathrm{~km}^{2}$ & \\
\hline Population & 14268 & 15132 & 46,6 \\
\hline Densité de population & 18,9 & 17,4 & $+1,7$ \\
\hline $\begin{array}{l}\text { Evolution de la population totale 1968- } \\
2014\end{array}$ & $-25 \%$ & $-14,4 \%$ & $+4,3 \%$ \\
\hline $\begin{array}{l}\text { Evolution de la population totale } \\
\text { 1999-2014 }\end{array}$ & $-5,5 \%$ & $-0,3 \%$ & \\
\hline
\end{tabular}

${ }^{2}$ C'est une Société d'Intérêt Collectif Agricole de Bourbon l'Archambault) créée dans les années 60. Elle est le seul abattoir agréé pour l'abattage des viandes sous Signe Officiel de Qualité Agneau label rouge, Bœuf Charolais label rouge et Agneau du Bourbonnais IGP et Bœuf Charolais du Bourbonnais IGP. C'est également l'abattoir qui réalise l'abattage, la découpe et la commercialisation des viandes sous la marque Cœur de Terroir. Elle regroupe 115 éleveurs principalement situées dans le Bocage Bourbonnais. 


\begin{tabular}{|l|c|c|c|}
\hline \% retraités/population totale & 37,1 & 34,0 & 33,6 \\
\hline$\%$ secteur primaire/emploi total & 19,6 & 18,4 & 5,7 \\
\hline \% secteur secondaire/emploi total & 8,5 & 9,5 & 15,4 \\
\hline \% secteur tertiaire/emploi total & 71,9 & 72,1 & 78,9 \\
\hline Taux de chômage 15-64 ans & 13 & 8,5 & 12,3 \\
\hline
\end{tabular}

Tableau 2: Inventaire des divers produits d'élevage sous signes de qualité présents sur les terrains d'étude (Chambre d'Agriculture, 2015)

\begin{tabular}{|c|c|c|}
\hline Démarches Qualité & Bocage Bourbonnais & Planèze de Saint-Flour \\
\hline $\mathrm{AOP}$ & & $\begin{array}{c}\text { Fromages Cantal*, Bleu } \\
\text { d'Auvergne*, Salers* }\end{array}$ \\
\hline Label Rouge et IGP & $\begin{array}{c}\text { Bœuf Charolais du Bourbonnais* } \\
\text { Agneau Fermier du } \\
\text { Bourbonnais* } \\
\text { Porc fermier d'Auvergne* } \\
\text { Volailles fermières d'Auvergne }\end{array}$ & $\begin{array}{l}\text { Porc fermier d'Auvergne } \\
\text { Salaisons d'Auvergne* }\end{array}$ \\
\hline Label Rouge & Tendre Agneau & $\begin{array}{c}\text { Viande Salers* } \\
\text { Bœuf Fermier d'Aubrac } \\
\text { Fleur d'Aubrac }\end{array}$ \\
\hline Agriculture Biologique & $\begin{array}{l}\text { Bœuf Charolais du Bourbonnais } \\
\text { Agneau Fermier du Bourbonnais } \\
\text { Porc fermier d'Auvergne }\end{array}$ & $\begin{array}{c}\text { Cantal } \\
\text { Tomme de montagne }\end{array}$ \\
\hline $\begin{array}{c}\text { Mention valorisante } \ll \text { produit de } \\
\text { montagne }\end{array}$ & & $\begin{array}{l}\text { Porc cantalou et capellin de } \\
\text { montagne } \\
\text { Mont lait } \\
\text { Les viandes du pays Vert }\end{array}$ \\
\hline En cours de demande AOP & & $\begin{array}{l}\text { Lentille blonde et pois blond de } \\
\text { Saint-Flour* }\end{array}$ \\
\hline $\begin{array}{l}\text { Sans labels ni mention } \\
\text { valorisante }\end{array}$ & $\begin{array}{l}\text { Produits laitiers* } \\
\text { Bières artisanales }\end{array}$ & $\begin{array}{c}\text { Seigle de Margeride } \\
\text { Cantal Haut Herbage } \\
\text { Pomme de Terre de Villedieu } \\
\text { Tripoux }\end{array}$ \\
\hline
\end{tabular}

Italiques: Zone de production limitée aux territoires étudiés ou leur proximité immédiate (quelques communes). * : Produits commercialisés dans les drives.

Ces territoires concentrent un grand nombre d'élevages mais la différence topographique des terrains entraine une conduite d'élevage différente et donc des types de productions distinctes. L'agriculture est fortement marquée par l'élevage herbivore qui concerne plus de $80 \%$ des exploitations agricoles sur ces deux terrains (Recensement Agricole, 2010). La SAU et le nombre d'UGB (Unité Gros Bétail) sont bien plus élevés dans le Bocage Bourbonnais (122 ha et 110 UGB) que sur la Planèze de Saint-Flour (72 ha et 68 UGB). D'après la typologie INOSYS $^{3}$ (INnOvation-SYStème) (Tableau 3), le Bocage Bourbonnais affiche un ensemble de huit types d'exploitations d'élevage dont les élevages bovins viande spécialisés représentent tout de même plus de $50 \%$ de ces exploitations. Arrivent ensuite les élevages ovins viande représentant $14 \%$ des exploitations, les polycultures viande (12\%) et les mixtes viande (bovins-ovins) avec 10\%. Le Bocage Bourbonnais est donc caractérisé par un terrain d'élevage de type allaitant. Pour la Planèze de Saint Flour/Margeride, cinq types

\footnotetext{
${ }^{3}$ Un outil permettant la construction d'une typologie des exploitations agricoles. Il a été construit à dires d'experts et éprouvé sur la base de données du Recensement Agricole 2010.
} 
d'exploitations ont été recensés mais trois systèmes dominent: les bovins-lait spécialisés (37\%), les mixtes lait-viande (35\%) et les bovins viande (24\%). La Planèze est une zone de convergence de différents types d'élevages avec une prédominance des troupeaux laitiers. Très peu d'exploitations sont en circuits courts par rapport à la moyenne régionale (19\%) : $9 \%$ dans le Bocage, $7 \%$ à Saint-Flour.

Tableau 3 : Typologie INOSYS des exploitations agricoles en Bocage Bourbonnais et Planèze de Saint-Flour (Gendron, 2016) (\%)

\begin{tabular}{|c|c|c|}
\hline Type d'exploitation & Bocage Bourbonnais & $\begin{array}{c}\text { Planèze de Saint Flour/ } \\
\text { Margeride }\end{array}$ \\
\hline Ovins viande spécialisé & 14 & 3 \\
\hline Bovins viande spécialisé & 50 & 24 \\
\hline $\begin{array}{c}\text { Petits ruminants lait } \\
\text { spécialisé }\end{array}$ & 2 & 0 \\
\hline Bovins lait spécialisé & 4 & 37 \\
\hline Mixtes viande & 10 & 1 \\
\hline mixtes lait-viande & 4 & 35 \\
\hline Polyculteurs lait & 4 & 0 \\
\hline Polyculteurs viande & 12 & 100 \\
\hline Total & 100 & \\
\hline
\end{tabular}

La démarche retenue comporte des enquêtes consommateurs auprès de la population locale et des entretiens qualitatifs menés auprès d'acteurs publics et privés.

\section{a. Les enquêtes consommateurs}

Afin de déterminer la composition potentielle d'un panier de biens, les préférences des consommateurs ont été identifiées, ainsi que leur CAP et leur degré d'attachement par rapport au territoire et à l'environnement. Deux types de questionnaires consommateurs ont été administrés :

- Le premier a bénéficié d'une diffusion en ligne auprès des clients des drives fermiers de l'Allier et de Saint-Flour ${ }^{4}$, il présente l'avantage d'accéder à un grand nombre de réponses dans un délai court. Les drives fermiers ont été choisis car ils permettent d'identifier directement une partie de la population résidente particulièrement sensible aux questions de localisation de la consommation. Ce choix se justifie également par la construction d'un vivier de consommateurs au sein duquel il est plus probable d'identifier un éventuel panier de biens complexes territorialisés. De plus, le mode de commercialisation des drives fermiers associe de facto plusieurs biens génériques et spécifiques. Les drives ne proposent qu'une partie limitée des produits spécifiques locaux (voir tableau 2).

- Le second a été effectué en face à face auprès d'un échantillon de 42 consommateurs questionnés au hasard sur le territoire du Bocage Bourbonnais particulièrement à la sortie de la Sicaba, du drive fermier, des grandes et petites surfaces et des marchés hebdomadaires. Ainsi, il a visé la caractérisation des habitués des drives fermiers parmi l'ensemble des consommateurs de produits locaux et plus largement les habitudes et sensibilités des consommateurs locaux.

\footnotetext{
${ }^{4}$ Pour ces deux drives, nous avons reçu respectivement 32 et 54 questionnaires remplis.
} 
Malgré leur différence notable, les deux questionnaires poursuivaient les mêmes objectifs suivants : Caractériser les consommateurs et leurs préférences pour des produits locaux issus de la diversité des systèmes d'élevages, identifier un panier de biens relatif à ces produits, approcher le consentement à payer du consommateur pour des produits locaux issus de la diversité de l'élevage et enfin déterminer la sensibilité environnementale et territoriale du consommateur. Le second questionnaire peut être perçu comme étant complémentaire au premier sur le terrain du Bocage Bourbonnais. Ainsi, par la suite «l'échantillon des deux drives fermiers » fera référence au premier questionnaire et «l'échantillon complémentaire » fera référence au second questionnaire.

\section{b. Les entretiens qualitatifs}

Ils ont été menés auprès d'acteurs (entreprises, collectivités, chambre d'agriculture, etc.) afin d'apprécier leurs stratégies mises en place et leur degré de coordination. Au total, 17 acteurs ont été interviewés sur l'ensemble des deux terrains, dont 11 sont rattachés à une activité de commercialisation (drive fermier, coopératives magasin de producteurs, bouchers) et 6 sont des acteurs publics (collectivités, lycée agricole). Néanmoins, la majorité des personnes enquêtées sont des acteurs de la commercialisation, qui ont une bonne connaissance des filières et des attentes des consommateurs. Les entretiens ont été de type semi-directif pour permettre une discussion ouverte et libre mais cadrée autour de trois axes : les produits du territoire et les canaux de distribution, les relations entre les acteurs des filières, et le comportement du consommateur. Les résultats des entretiens sont analysés de manière qualitative pour déterminer les modes d'organisation des acteurs, leurs coordinations et le mode de gouvernance. L'analyse des données a été faite à partir d'une grille d'analyse théorique centrée sur les déterminants suivants: l'ancrage territorial, la démarche du consommateur, le type de marché, la concurrence externe, la coordination au sein de la filière, inter-filières et le rôle des acteurs publics (Roux, Vollet et Pecqueur, 2006).

\section{Des modèles de biens complexes territorialisés très spécifiques aux consommateurs locaux}

Au total, 130 individus ont répondu aux deux questionnaires. L'échantillon se compose de consommateurs résidents ${ }^{5}$ dans les deux terrains d'étude avec $65 \%$ de femmes et $35 \%$ d'hommes représentant toutes les tranches d'âge. Parmi les catégories socioprofessionnelles les plus représentées, $32 \%$ sont des ouvriers ou employés (voir tableau 4).

Tableau 4 : Les caractéristiques des consommateurs

\begin{tabular}{|cc|c|c|c|}
\hline \multicolumn{2}{|l|}{ Caractéristiques } & $\boldsymbol{\%}^{\mathbf{6}}$ & Caractéristiques & \% \\
\hline Sexe & & Age & \\
& Hommes & 35 & {$[18-29]$} & 16 \\
& Femmes & 65 & {$[30-44]$} & 32 \\
\hline Age & & {$[45-59]$} & 28 \\
& {$[18-29]$} & 16 & {$[60$ et +$]$} & 24 \\
& {$[30-44]$} & 32 & & \\
& {$[45-59]$} & 28 & & \\
& {$[60$ et +] } & 24 & & \\
\hline
\end{tabular}

\footnotetext{
${ }^{5}$ Les consommateurs habitent dans un rayon de 30 à $50 \mathrm{Km}$ autour de Bourbon l'Archambault et Saint-Flour.

${ }^{6} \mathrm{C}$ 'est une moyenne pondérée des deux terrains.
} 


\begin{tabular}{|l|l|c|l|}
\hline Catégories socioprofessionnelles & & Revenu $(€)^{7}$ & \\
Agriculteurs & 12 & $>1000$ & 8 \\
Artisans, commerçants, chefs d'entreprise & 10 & {$[1000,2000]$} & 42 \\
Cadres ou professions intellectuelles supérieures & 9 & {$[2000,3000]$} & 29 \\
Professions intermédiaires & 8 & {$[3000,5000]$} & 18 \\
Ourriers, employés & 32 & $<5000$ & 3 \\
Sans activité professionnelle & 8 & & \\
Retraités & 19 & & \\
Etudiants & 2 & & \\
& & & \\
\end{tabular}

Les résultats obtenus tendent à mettre en évidence de véritables particularités dans la constitution des modèles de biens complexes territorialisés dans le cas des consommateurs locaux. Les biens constitutifs sont communs aux deux territoires (bœuf, porc et légumes). Les consommateurs sont d'autant plus enclins à reconnaître leur spécificité que de véritables coordinations organisationnelles se sont mises en œuvre pour les constituer en ressources territoriales. Les consommateurs ont déclaré des consentements à payer significativement supérieurs pour les produits locaux. Celui-ci est en fait beaucoup plus lié à la vitalité sociale qu'aux aménités paysagères.

\section{a. Vers l'identification d'un « panier moyen» bœuf-porc-légumes pour les résidents permanents des deux territoires}

$79 \%$ des consommateurs de l'échantillon achètent régulièrement de la viande de bœuf produite dans le département et $61 \%$ d'entre eux privilégient la race charolaise. Ils sont ensuite plus de la moitié (57\%) à acheter de la viande locale de porc qui provient d'un périmètre plus large que le Bocage mais qui reste auvergnate et de qualité avec la certification «Porc Fermier d'Auvergne ». Un peu moins de la moitié (41\%) d'entre eux achètent souvent des produits de maraichage en même temps que la viande locale (Tableau 5). Arrivent ensuite le fromage et les autres produits laitiers (28\%), puis la charcuterie pour 15\% des individus interrogés. Certains de ces produits (maraichage, œufs) apparaissent véritablement comme des «produits d'appel » car non seulement ce sont des produits de consommation courante mais aussi parce que leur présence sur un même lieu d'achat incite les consommateurs à acheter d'autres produits agricoles plus onéreux (produits laitiers, viande).

De plus, une complémentarité d'achat entre la viande bovine et la viande porcine apparait sur les deux terrains puisque pour plus de $50 \%$ des consommateurs des deux échantillons, il est important pour eux de pouvoir acheter ces deux viandes sur le même lieu d'achat au sein d'un même panier ${ }^{8}$ (Tableau 6). C'est également le cas pour la volaille mais dans des proportions plus faibles, notamment dans le Bocage Bourbonnais. Les viandes de veau et surtout d'agneau sont achetées simultanément dans des proportions bien plus faibles de l'ordre de 20 à $30 \%$ des consommateurs. Cependant, le lieu d'achat actuel de chaque viande est différent (Tableaux 7 et 8). Sur les deux terrains, les consommateurs enquêtés préfèrent acheter le porc dans les Grandes et Moyennes Surfaces (GMS). A contrario, ils cherchent le contact avec le

\footnotetext{
${ }^{7}$ La répartition des individus par rapport au revenu concerne uniquement le second questionnaire (42 consommateurs).

${ }^{8}$ Lors de la discussion des résultats, nous utiliserons le terme de «panier » pour designer toutes formes de biens complexes territorialisés afin de faciliter la rédaction et la compréhension. Dans le modèle du «panier », il existe une véritable complémentarité entre produits et services mais ce n'est pas toujours le cas. La complémentarité peut être que potentielle, sans synergie réelle entre eux, ni coordinations efficaces entre acteurs : on parle alors d'assortiment ou de «puzzle» (Mollard et al., 2005).
} 
producteur ou avec un professionnel lors de leurs achats de viande bovine. En effet, sur la planèze, $36 \%$ d'entre eux achètent la viande bovine en boucherie et $51 \%$ des consommateurs questionnés du Bocage privilégient les circuits courts comme la vente directe ou les magasins de producteurs ou drives fermiers. De plus, la présence d'une coopérative d'éleveurs, Sicaba, valorisant l'élevage du Bourbonnais procure une certaine spécificité au territoire par rapport à la Planèze. Elle propose une autre forme de circuits de distribution qui centralise $12 \%$ des achats de viande des consommateurs interrogés. Ainsi, elle a établi une relation de proximité et de confiance avec les consommateurs locaux grâce à son ancrage et sa réputation, comme cela a déjà été constaté dans les filières viande d'autres territoires (Margetic, 1999 ; Aubrée et al., 2018). Parallèlement, les fréquences d'achat sont donc différentes en fonction des lieux d'achat : la vente directe propose des formats d'achat avec de plus gros volumes comme des caissettes. Les achats sont donc moins fréquents dans le bocage, 1 à 3 fois par mois ou quelques fois par an, tandis que les achats à Saint-Flour sont plus réguliers.

Tableau 5 : Les principaux produits achetés en même temps que la viande bovine (Bocage Bourbonnais et Planèze de Saint-Flour)

\begin{tabular}{|c|c|}
\hline Produits & Pourcentage \\
\hline Légumes-fruits frais et secs & 44,4 \\
\hline Fromages et produits laitiers & 28,2 \\
\hline Charcuterie & 1,1 \\
\hline CEufs & 3,0 \\
\hline Boissons (alcoolisés ou non) & 10,4 \\
\hline
\end{tabular}

Tableau 6 : Pourcentage de consommateurs achetant les autres viandes en même temps que le bœuf (\%)

\begin{tabular}{|c|c|c|c|c|}
\hline & Agneau & Porc & Veau & Volaille \\
\hline Planèze (drive uniquement) & 33,3 & 75,4 & 49,1 & 68,4 \\
\hline Bocage (total consommateurs) & 19,1 & 52,4 & 29,3 & 45,3 \\
\hline Bocage (drive uniquement) & 22,5 & 61,2 & 41,9 & 54,8 \\
\hline
\end{tabular}


Tableau 7 : Répartition des lieux d'achat par type de viande sur la Planèze de Saint-Flour/Margeride (\%)

\begin{tabular}{|c|c|c|c|c|}
\hline & Vente directe & Boucherie & Magasins de producteurs, drive fermier, marché & Supermarchés \\
\hline Bœuf & 23,2 & 35,7 & 19,6 & 23,2 \\
\hline Agneau & 23,2 & 30,3 & 19,6 & 28,5 \\
\hline Porc & 12,5 & 23,2 & 28,5 & 37,5 \\
\hline Veau & 23,2 & 35,7 & 21,4 & 21,4 \\
\hline
\end{tabular}

Tableau 8 : Répartition des lieux d'achat par type de viande dans le Bocage Bourbonnais (\%)

\begin{tabular}{|c|c|c|c|c|c|}
\hline & $\begin{array}{c}\text { Vente } \\
\text { directe }\end{array}$ & Boucherie & $\begin{array}{c}\text { Magasins de producteurs, drive fermier, } \\
\text { marché }\end{array}$ & Supermarchés & Sicaba \\
\hline Bœuf & 38,1 & 20,8 & 25,0 & 18,5 & 35,7 \\
\hline Agneau & 14,2 & 29,2 & 21,8 & 29,6 & 28,6 \\
\hline Porc & 28,6 & 20,8 & 25,1 & 29,6 & 14,2 \\
\hline Veau & 19,1 & 29,2 & 28,1 & 22,2 & 21,4 \\
\hline
\end{tabular}

Les viandes d'agneau et de veau semblent être consommées dans des proportions moindres, mais sensiblement plus que la moyenne nationale (Tableau 9). Ces données reflètent la tendance générale. Effectivement, en France, la viande la plus consommée, y compris sous forme transformée, est la viande porcine. Le porc bénéficie d'un prix peu élevé par rapport aux autres viandes et présente une importante diversité de produits ce qui peut expliquer cette tendance. La viande bovine, est la troisième viande la plus consommée après la volaille, mais qui est en baisse depuis 2004 (-2,5kg de moins). La viande d'agneau est très peu consommée ( $3 \mathrm{~kg} / \mathrm{an} /$ personne), en raison de son niveau de prix élevé et de son gout prononcé. On peut tout de même noter cette ambivalence entre le fait que les consommateurs enquêtés du Bourbonnais estiment la viande d'agneau comme emblématique du territoire mais la consomment très peu par rapport aux autres viandes, même moins que l'échantillon de la Planèze.

Tableau 9 : Récapitulatif des quantités achetées par type de viande en kilos par habitant et par an, sur les deux terrains d'étude et à échelle nationale

\begin{tabular}{|l|c|c|c|c|}
\hline & $\begin{array}{c}\text { Quantité } \\
\text { consommée de } \\
\text { bœuf (kg/par } \\
\text { hab./an) }\end{array}$ & $\begin{array}{c}\text { Quantité } \\
\text { consommée de } \\
\text { porc (kg/par } \\
\text { hab./an) }\end{array}$ & $\begin{array}{c}\text { Quantité } \\
\text { consommée } \\
\text { d'agneau (kg/par } \\
\text { hab./an) }\end{array}$ & $\begin{array}{c}\text { Quantité } \\
\text { consommée de } \\
\text { veau (kg/par } \\
\text { hab. /an) }\end{array}$ \\
\hline Planèze Saint-Flour & 28,1 & 31,6 & 12,5 & 16,9 \\
\hline Bocage Bourbonnais & 26,1 & 29,3 & 7,1 & 12,36 \\
\hline National (Interbev, 2014) & 21,6 & 28,8 & 3,0 & 3,7 \\
\hline
\end{tabular}

Lors de leurs achats de viande, $70 \%$ des consommateurs de l'échantillon total privilégient l'origine locale (Figure 2) en favorisant ainsi leur territoire. Ensuite, sur Saint-Flour, presque la moitié des sondés se renseignent par rapport au prix (39\%) et à l'aspect physique du produit 
(36\%). A l'inverse, dans le Bocage, un mode de production respectueux de l'environnement est recherché pour $70 \%$ des consommateurs enquêtés. Ce critère d'achat est tout aussi déterminant que l'origine de production. Cette disparité entre les deux échantillons peut s'expliquer par une sensibilité globale plus forte et ancienne par rapport aux enjeux environnementaux dans l'Allier. Cette sensibilité se traduit dans les politiques territoriales mises en œuvre : la communauté de communes a mis en place un projet territorial «bio-éco » qui consiste à accompagner l'installation d'acteurs «bioécologiques » toutes activités confondues (agricole, artisanale, commerciale). Un volet consacré à la communication sur cette thématique est également conduit. Toutes ces initiatives ont pu influencer la population locale dans leurs comportements d'achat. De plus, la proximité avec un pôle urbain, Moulins (situé à une vingtaine de kilomètres), a pu aussi accroitre leur sensibilité aux enjeux environnementaux.

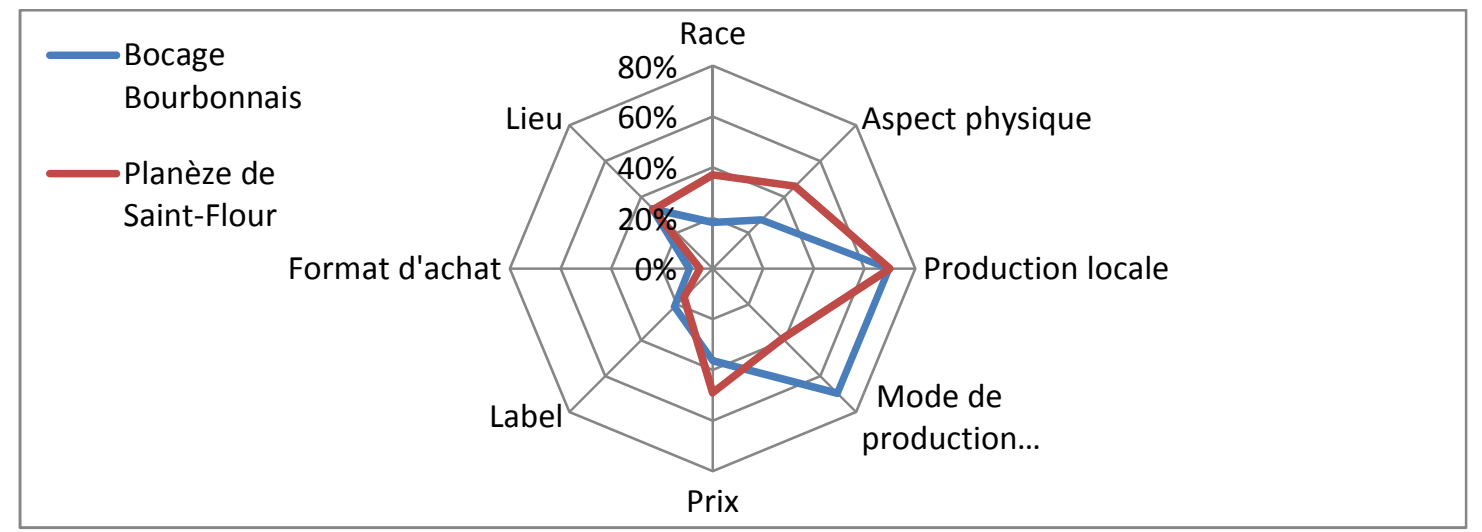

Figure 2 : Critères de choix d'achat des consommateurs de la Planèze de Saint-Flour et du Bocage Bourbonnais

\section{b. Une typicité des produits, identifiée par les consommateurs, très dépendante de la construction des ressources territoriales}

Les consommateurs questionnés du drive de la Planèze de Saint-Flour ont jugé la lentille blonde de Saint-Flour et les charcuteries et salaisons comme étant les productions les plus emblématiques du territoire. Ils choisissent ensuite, la viande bovine et porcine et en dernière position le seigle de Margeride et la viande d'agneau (Tableau 10). Les entretiens menés avec les responsables de l'association des producteurs de lentille blonde de Saint-Flour ont révélé qu'actuellement, la lentille blonde de Saint-Flour fait l'objet d'une reconstruction territoriale au niveau de son image et de son histoire grâce à l'efficacité d'une organisation locale collective, soutenue par les acteurs publics des collectivités et du tourisme, qui a joué un rôle important dans la dynamique de construction, comme cela a été constaté dans d'autres contextes géographiques (François et al., 2006). La production de lentille a été relancée en 2006, après des années de recherche autour de la semence qui avait disparu au profit de l'intensification des élevages laitiers. Suite à la dernière crise laitière, une nouvelle dynamique s'est installée en créant une filière et en reconstruisant une image haut de gamme à cette légumineuse. Les habitants ont donc pu voir éclore cette reconstruction et se sont appropriés ce produit. En revanche, cette production reste minoritaire puisqu'elle est cultivée sur 80 ha et sa production est très variable d'une année à l'autre, de 40 à 70 tonnes. La culture de lentille reste un moyen de diversification pour pallier la crise laitière. Dans la continuité de la dynamique, la fédération d'agriculteurs construit un dossier pour une demande d'AOC avec 
tout de même un point critique que relève l'INAO sur l'histoire et la disparition de la semence pendant un certain temps.

Tableau 10 : Classement des produits de la Planèze de Saint-Flour selon leur typicité d'après les consommateurs enquêtés

\begin{tabular}{|l|c|c|}
\hline & Planèze de Saint Flour & Bocage Bourbonnais \\
\hline $\mathbf{1}$ & Lentille & Bœuf \\
\hline $\mathbf{2}$ & Charcuterie & Veau \\
\hline $\mathbf{3}$ & Bœuf Aubrac & Agneau \\
\hline $\mathbf{4}$ & Porc & Porc \\
\hline $\mathbf{5}$ & Bœuf Salers & Charcuterie et salaisons \\
\hline $\mathbf{6}$ & Cantal & Fromages \\
\hline $\mathbf{7}$ & Seigle de Margeride & $/$ \\
\hline $\mathbf{8}$ & Agneau & $/$ \\
\hline
\end{tabular}

A l'opposé, en Margeride dès le XVI ${ }^{\text {ième }}$ siècle, la tradition agricole reposait sur un système agro-pastoral associant la culture de seigle et l'élevage d'ovins. Dès les années 30, le faible rendement et donc la faible rentabilité de ce système a entrainé le déclin de cette tradition. La production de seigle est actuellement assurée par une seule entreprise «Les moulins d'Antoine» qui instaure des quotas de production. L'absence d'organisation et la gouvernance privée d'une seule entreprise sur la production ne permet pas une valorisation efficiente et un ancrage identitaire fort. On notera l'ambiguïté autour du fromage Cantal, nonconsidéré par le panel comme un produit emblématique de la Planèze, pourtant consacrée à la production laitière depuis les années 50 . La structuration de la filière et l'obtention d'un sigle officiel de qualité, AOC, pourraient pourtant laisser présager le contraire. Mais la large gamme de produits présents sur le cantal (jeune, entre-deux et vieux), un cahier des charges peu restrictif dans une zone large et des conflits au sein même de la filière entre les différents acteurs tendent à perdre le consommateur qui peine à trouver une unité et une identité au produit.

Quant à l'échantillon des consommateurs du Bocage Bourbonnais, ils déclarent que les viandes du Bourbonnais font la spécificité du territoire avec très largement en tête la viande bovine, puis le veau, l'agneau et le porc (Tableau 10). Ces premières observations laissent présager que les consommateurs enquêtés ont conscience de ce qui fait l'identité du Bourbonnais, c'est-à-dire l'élevage herbivore. La zone de production du bœuf et de l'agneau du Bourbonnais est délimitée par l'apposition d'un signe officiel de qualité, IGP. L'INAO définit l'IGP comme un signe officiel qui garantit au consommateur la qualité, la réputation ou les caractéristiques intrinsèques de ces produits en lien avec leur origine géographique. Les fromages, de vache, de chèvre ou de brebis, sont considérés comme une production standard et non spécifique par le panel. En effet, cette production est plutôt marginale sur le territoire et réalisée directement à la ferme. Les consommateurs interrogés ne perçoivent pas de spécificité liée à une origine géographique, une race spécifique ou un savoir-faire particulier. La place des charcuteries et salaisons est très différente selon les territoires. En effet, ces produits semblent emblématiques sur la Planèze alors que dans le Bocage Bourbonnais ils ne sont que très peu reconnus. Cette distinction peut s'expliquer par la tradition laitière d'une partie du département du Cantal où le petit lait issu de la transformation fromagère était donné aux porcs (Tardieu et Rudelle, 1962). Bien que cette pratique ait beaucoup régressé, elle reste très présente dans les représentations des acteurs locaux, y compris publics. On peut citer la volonté de la communauté de communes de Saint-Flour de soutenir la filière porcs nourris à 
base de petit lait. Par ailleurs, la présence d'un atelier de découpe et de transformation au lycée agricole de Saint-Flour suite à un investissement de la communauté de communes, accentue l'ancrage de la filière porc (viande fraiche et produits de salaisons). Le maintien des abattoirs de proximité sur le territoire et l'implantation d'un atelier de découpe et de transformation a permis le développement de la transformation charcutière. De plus, les politiques de valorisation mises en place dans le Cantal sont orientées vers les produits de montagne dont font partie les charcuteries et salaisons. De cette manière, les consommateurs locaux sont plus sensibilisés à cette production locale.

\section{c. Des « CAP » significativement supérieurs pour les produits locaux des 2 territoires}

Sur les deux terrains, les consommateurs interviewés des drives fermiers semblent être prêts à payer plus cher un produit local issu d'un circuit court (Tableau 11), corroborant ainsi les résultats obtenus par Hérault-Fournier (2012) sur le rôle des proximités identitaire et relationnelles dans le cas de circuits courts alimentaires. Dans les deux territoires et sur une échelle de 1(très élevé) à 7 (très faible), le CAP pour un produit local parait élevé puisque les consommateurs enquêtés jugent le prix faible (ce qui est cohérent avec le survey de Fledmann et Hamm, 2015 : une majorité d'études consommateurs tendent à montrer que les prix des produits locaux ne sont pas perçus comme chers par les consommateurs). En revanche, pour des produits certifiés dans le cas d'une production bovine, les enquêtés semblent avoir un CAP faible.

Tableau 11 : Moyennes des CAP selon les critères choisis par terrain d'étude

\begin{tabular}{|c|c|c|c|c|}
\hline & CAP Race & CAP label & CAP bio & CAP circuit court \\
\hline Bocage Bourbonnais & 5,5 & 4,8 & 4,9 & 3,0 \\
\hline Planèze de Saint-Flour & 5,0 & 4,3 & 4,7 & 2,8 \\
\hline
\end{tabular}

Pour l'ensemble de l'échantillon du Bocage Bourbonnais, les résultats sont identiques ; plus des trois-quarts des consommateurs interrogés sont prêts à acheter un produit local plus cher qu'un autre. La plupart d'entre eux payeraient $5 \%$ ou $10 \%$ de plus pour acquérir un produit local. Toutefois, peu d'entre eux seraient prêts à dépasser une différence de $15 \%$.

Le CAP peut présenter une variabilité interindividuelle et interproduit forte. A titre d'exemple, des questions spécifiques ont été posées aux consommateurs de l'échantillon complémentaire du Bocage lors de l'achat de steaks de bœuf (Figure 3). Les résultats montrent que lorsque l'on propose aux consommateurs de choisir entre un steak de bœuf Charolais Standard, LR ou Bio, tous trois provenant de la SICABA, 55\% des consommateurs préfèrent acheter la qualité Label Rouge, coûtant $19 € / \mathrm{kg}^{9}$ puis $27,5 \%$ préfèrent la qualité Standard $(17 € / \mathrm{kg})$. La viande charolaise de qualité standard de la Sicaba est plus chère qu'en GMS ( $3 €$ de plus le kilo), ce qui prouve que les consommateurs interrogés qui l'achètent dans cette boucherie recherchent quelque chose qu'ils ne trouveraient pas dans une GMS ; il s'agit de la qualité de la viande mais aussi de la confiance qu'ils ont dans le produit grâce à la transparence et la proximité de la chaine de production. De même, ils sont conseillés par des personnes qualifiées qui sont en mesure de fournir aux consommateurs des conseils spécifiques. Enfin, pour une viande de qualité Bio, les consommateurs interrogés ont tendance à privilégier des lieux d'achat qui leur procureraient une meilleure «confiance »: ils sont autant à choisir le Drive fermier que la Sicaba (19 consommateurs d'un côté et 18 de l'autre). Les deux points de vente proposent le steak de bœuf Bio au même prix $(18 € / \mathrm{kg})$.

\footnotetext{
${ }^{9}$ Tous les prix ont été relevés sur les différents points de vente en septembre 2017.
} 


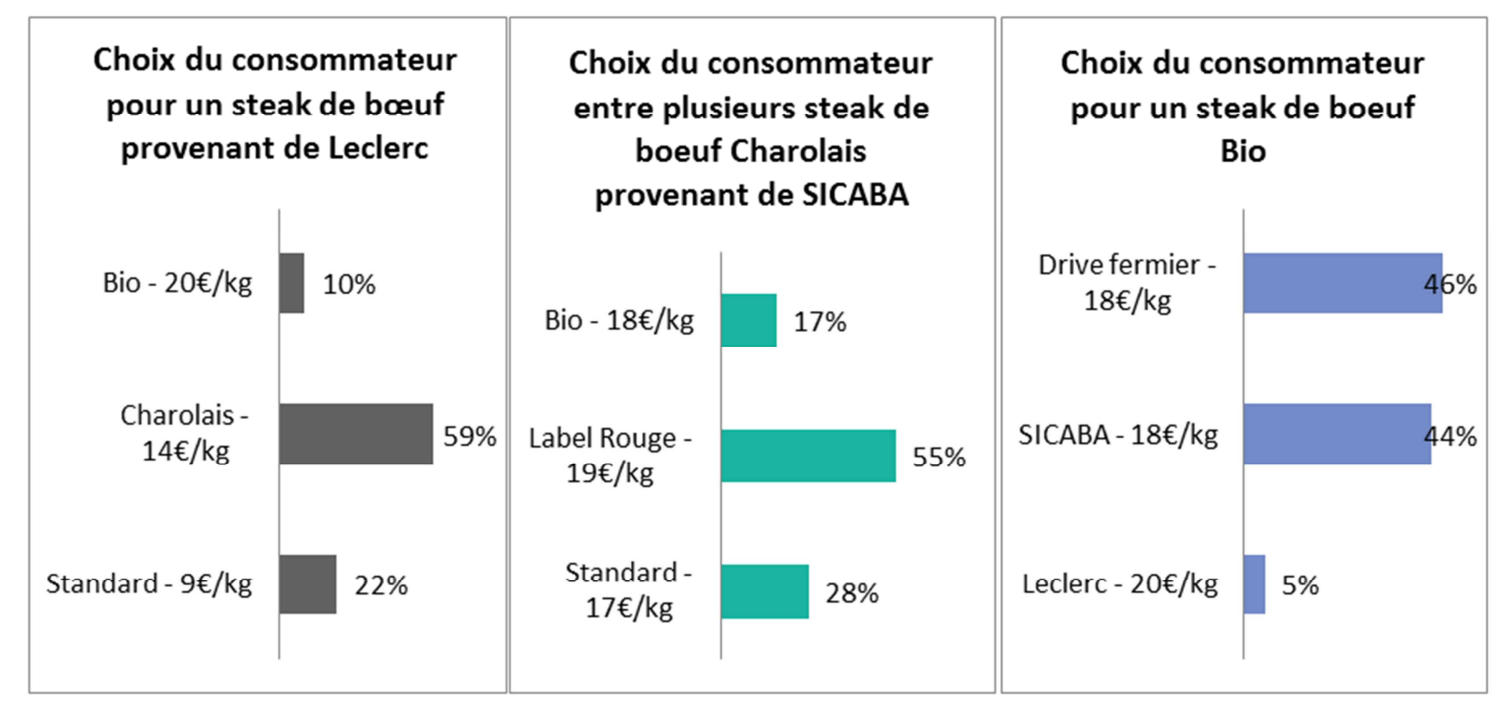

La somme des pourcentages des graphiques Q13.1 et Q13.3n'est pas égale à 100\% car certains consommateurs ont refusé de répondre à la question, car ce mode d'achat serait " contraire à leurs valeurs ».

Figure 3 : Comparaison des choix des consommateurs entre diverses qualités de steak de bœuf selon le lieu d'achat

Les motivations d'achat des consommateurs relatifs aux deux enquêtes pour les produits locaux sont de plusieurs ordres : il s'agit d'abord d'une certaine confiance à l'égard du producteur local (62,5\% ont placé ce critère dans les trois premières positions du classement), vient ensuite la qualité du produit $(41 \%)$. D'autres raisons, telles que la contribution à l'activité socio-économique du territoire, l'origine, le goût et la traçabilité du produit ont aussi été évoquées par les répondants. Cette préférence forte et croissante pour les produits locaux des 2 territoires pourrait s'expliquer par la recherche de confiance, qui se traduirait par une évolution forte des consommateurs ces 5 dernières années. En effet, dans le second questionnaire, près de $80 \%$ d'entre eux déclarent acheter davantage de produits locaux en cherchant à connaitre plus l'origine du produit avant l'achat et en privilégiant les produits issus de l'agriculture française et ce malgré le faible nombre d'exploitations tournées vers les circuits courts. Ils restent très sensibles au signal prix puisque près de $40 \%$ d'entre eux déclarent qu'ils achèteraient davantage de produits locaux si les prix étaient plus faibles, ce qui représentent le pourcentage le plus élevé parmi les facteurs évoqués (Figure 4). 


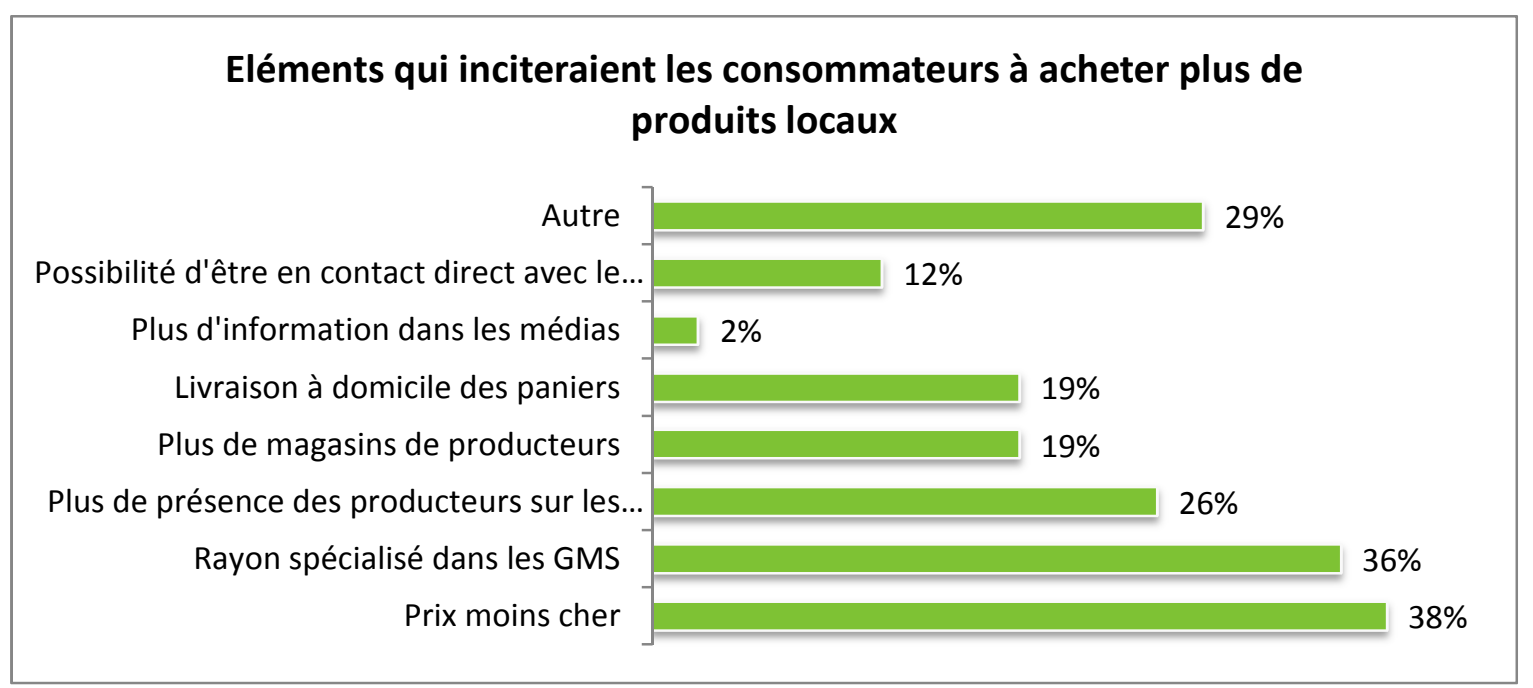

Figure 4 : Les principaux éléments pouvant inciter à l'achat de produits locaux

\section{d. Un ensemble de services joints aux biens complexes territorialisés liés à la vitalité sociale plus qu'aux aménités paysagères}

Au total, nous disposons d'indices concordant nous permettant d'affirmer qu'il existe dans les deux territoires des types de «panier» par rapport à la consommation locale : achat simultanée de produits et CAP plus fort pour les produits locaux. Toutefois, ces modèles présentent des caractéristiques, dont certaines peuvent à terme compromettre leurs dynamiques à moyen terme.

Contrairement à la plupart des paniers de biens mis en évidence pour la clientèle touristique, les consommateurs locaux interrogés sont faiblement sensibles aux ressources paysagères. En effet, ils sont seulement $14 \%$ à penser qu'en achetant des produits locaux ils contribuent au maintien des ressources paysagères (question posée en proposant une diversité de réponses) De même, lorsqu'on leur demande pour quelles raisons ils achètent des produits locaux, ils sont plus de $21 \%$ à avoir cité le fait de contribuer à l'entretien du paysage bourbonnais. Ces résultats tendent à montrer que les consommateurs interrogés ne perçoivent qu'une très faible relation (si ce n'est pas du tout) entre les produits locaux issus de l'élevage et les biens produits conjointement avec cette activité agricole.

En revanche, l'échantillon interrogé effectue un lien fort entre l'achat de produits locaux et le maintien de l'emploi local. Plus de $75 \%$ d'entre eux mettent en avant ce type d'argument comme motif majeur d'achat (Figure 5). D'autre part, environ un tiers des consommateurs du Bocage Bourbonnais et un peu de la moitié de ceux de la Planèze de Saint-Flour pratiquent des activités culturelles ou festives localement. Cette sensibilité à la vitalité sociale est peut être liée à la diminution continue de la population totale sur les deux zones en longue période alors que ce n'est pas forcément le cas de l'ensemble du massif central (où certaines parties du massif plus proches des villes ou plus méridionales connaissent des évolutions plus favorables). Le niveau du chômage, plus élevé (dans le Bocage Bourbonnais) peut également constituer un des éléments explicatifs de contexte (voir Tableau 1).

Les services liés au panier ou de façon plus générale au modèle de biens complexes territorialisés n'est pas forcément naturel (de type services non marchands paysagers) mais plutôt de type social, c'est-à-dire une utilité liée à la vitalité territoriale (emploi local, animations culturelles et festives). Dans les territoires où l'élevage est fortement présent, les 
services liés à la vitalité sociale ont été déjà identifiés par d'autres auteurs (Ryschawy, 2015). Nos résultats tendent à montrer qu'ils sont surtout attendus par les consommateurs locaux.

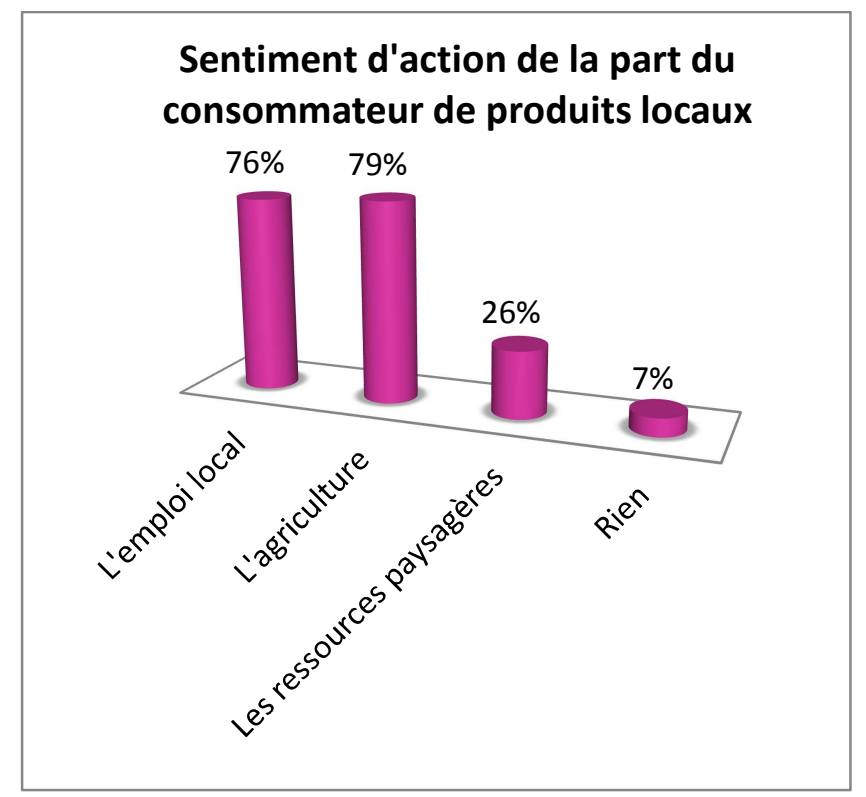

Figure 5 : Les principales motivations d'achat mises en avant par l'ensemble des consommateurs interrogés (Bocage Bourbonnais) lors de l'achat de produits locaux

Dans cet ensemble de services joints aux biens complexes territorialisés, la biodiversité des animaux domestiques (bovins) tient une place très particulière. Le lien entre l'achat de la viande bovine et les races locales est en effet difficile à décrypter. Dans le Bocage Bourbonnais, $61 \%$ des consommateurs achètent de la viande de bœuf de race Charolaise, mais un quart de l'échantillon ne tient pas compte de la race lors de l'achat de viande. De surcroît, $15 \%$ des individus interrogés préfèrent des races non liées à la zone (Aubrac ou Limousine) (Figure 6). Pour les consommateurs du drive fermier, dans une GMS, plus de la moitié des consommateurs préfèrent acheter un steak de bœuf de race Charolaise $(58,5 \%)$ plutôt que du bœuf standard $(22 \%)^{10}$ alors que le prix au kilo du premier coûte $5 €$ de plus que celui du second.

\footnotetext{
${ }^{10}$ Ils ne sont que $10 \%$ à préférer acheter de la qualité Bio dans une GMS.
} 


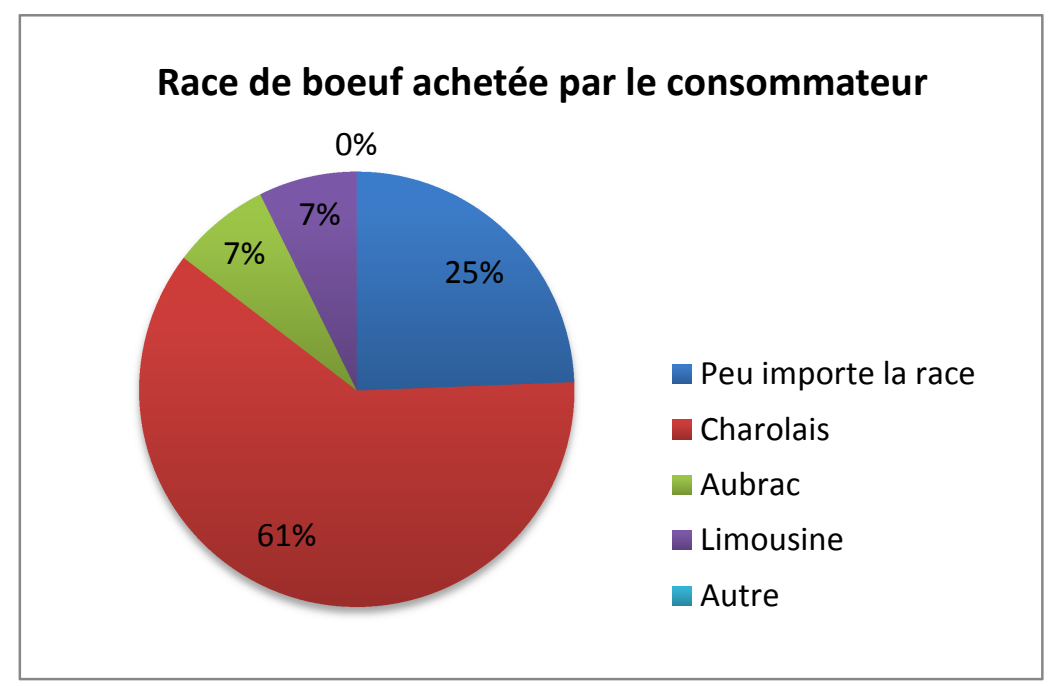

Figure 6 : Race de bœuf acheté par le consommateur dans le Bocage Bourbonnais

\section{Conclusion}

Cette étude puise son originalité dans différents facteurs. Les territoires d'étude se caractérisent par la diversité des produits d'élevage engendrant une diversité de stratégies de valorisation. L'élevage herbivore étant l'activité dominante de l'agriculture sur ces terrains, la viande apparait donc comme le produit phare. Le modèle de biens complexes territorialisés est un cadre d'analyse qui permet de soulever les questions de valorisation de cette diversité de produits liés à l'élevage. La place de la viande au sein de ce «panier de biens » est abordée de manière originale ainsi que son rôle dans la valorisation des ressources territoriales. Par ailleurs, le modèle de biens complexes est appliqué à deux territoires qui ne sont pas reconnus comme des zones touristiques. Ainsi, la valorisation des produits est considérée dans ce travail uniquement sur la base d'une consommation locale.

D'après les résultats des enquêtes consommateurs et des entretiens réalisés auprès des acteurs des deux territoires étudiés, l'hypothèse sur la possibilité de stratégie de biens complexes territorialisés autour d'une consommation locale de produits issus de l'élevage est plutôt validée. Dans ce travail, les analyses ont permis d'identifier un «panier moyen » bœuf-porclégume pour les résidents des deux territoires. Contrairement aux modèles de biens complexes territorialisés mis en évidence dans le cas des touristes (Roux et al., 2007), certains produits périssables comme la viande peuvent être intégrés dans une stratégie de biens complexes territorialisés ou de panier et les productions fromagères jouent un rôle mineur. Pour cette raison, les rapports homme/produits/territoire déjà explorés par ailleurs (Frayssignes, 2008 ; Delfosse, 2006) sont manifestement de nature très différente dans le cas de consommation touristique ou de consommation locale. En effet, la dimension paysagère pour les services non marchands ainsi que la praticité des produits jouent un rôle majeur dans la consommation touristique. En revanche pour la consommation locale, les aspects de vitalité territoriale (emplois mais aussi animations culturelles) constituent des caractéristiques importantes. Cependant, la spécificité des produits est un aspect incontournable dans les deux cas, probablement de façon moins prononcée pour la consommation locale mais ce point reste probablement à confirmer. Cependant, l'identification des produits typiques de chaque zone d'étude est très dépendante de la construction des ressources territoriales et des actions de coordinations locales. Certaines filières se sont caractérisées par leur efficacité pour construire, voire même dans certains cas reconstruire une spécificité forte et reconnue par les consommateurs (comme la lentille blonde sur la Planèze) alors que d'autres filières pâtissent 
véritablement d'un manque de coordinations (seigle de Margeride notamment). En revanche pour les deux territoires, les CAP sont significativement supérieurs pour des produits locaux dénotant un ensemble de services joints qui est liée à la vitalité sociale plus qu'aux aménités paysagères.

Le travail présenté ici reste exploratoire et il faudrait le confirmer par des études plus quantitatives analysant les comportements d'achat de divers types de consommateurs (certains travaux antérieurs ayant clairement montré que l'appartenance des producteurs et des consommateurs locaux à une même classe sociale favorise la performance des circuits courts : Richard et al., 2014). Cependant, la pérennité de ce modèle dépend de l'existence d'un certain nombre de conditions liées aux produits et à la gouvernance pour induire ou susciter des ventes de produits issus des systèmes d'élevage et aux circuits courts (Aubree et al., 2018). Bien qu'ils n'aient pas de tradition, ou d'ancrage territorial particulier, sur les deux terrains (sauf à intégrer leurs marges comme la vallée de l'Allagnon pour la Planèze de Saint-Flour), les fruits et légumes représentent des produits d'appel même si la filière n'est que très peu structurée sur la Planèze, à cause des raisons topographiques. Quant aux autres produits composant le «panier » potentiel, ce sont les mêmes sur les deux territoires, la viande bovine et porcine (fraiche ou transformée) s'appuyant sur des caractéristiques de production et des modes d'organisation propres à chacun des territoires : une filière qualité bovine dans le Bocage structurée autour de la Sicaba et des caractéristiques raciales avec un fort ancrage territorial pour la Planèze. Ces deux points renforcent respectivement l'ancrage territorial de chaque filière que ce soit par l'intermédiaire d'un acteur privé ou par les caractéristiques intrinsèques du produit afin d'en faire un produit leader du «panier». Enfin, la viande porcine trouve également sa place parmi les candidats au panier potentiel puisque c'est un produit apprécié des consommateurs, sous sa forme fraiche et transformée. Ayant plus de typicité sur la Planèze et issue d'une production traditionnelle, les acteurs publics ont une réelle volonté de développer une filière locale structurée, ce qui pourrait ouvrir des perspectives de développement pour cette filière en devenir et qu'une analyse en termes de capabilités telle que celle développée par Chiffoleau et Prévost (2012) pour les circuits courts permette de rendre compte.

\section{Références}

ANGEON V., VOLLET D., 2008, Spécification des produits, asymétries d'information et développement territorial. Le paradoxe de la filière coutelière dans l'Aubrac, Revue d'Economie Régionale et Urbaine, 591-616.

Aubrée, P., Chiffoleau, Y., Villarroël, A. 2018 Coopération entre agriculteurs et artisans commerçants dans les circuits alimentaires de proximité. Innovations Agronomiques, 63, $57-$ 69.

Bergadaa M. et Del Bucchia C. 2009, La recherche de proximité par le client dans le secteur de la grande consommation alimentaire, Management \& Avenir, Vol. 21, 121-135.

CHIFFOLEAU Y., PREVOST B. 2012, Les circuits courts, des innovations sociales pour une alimentation durable dans les territoires, Norois, 224, 7-20.

DELFOSSE C., 2006, La localisation de la production fromagère : évolutions des approches géographiques, Géocarrefour, 81(4), 311-318.

FRANÇOIS H., HIRCZAK M., SENIL N., 2006, Territoire et patrimoine : la co-construction d'une dynamique et de ses ressources, Revue d'Economie Régionale et Urbaine, 5, 683-700. 
FRAYSSIGNES J., 2008, Démarches de qualité et développement territorial: quels apports pour la géographie rurale ? L'exemple des AOC fromagères françaises, Géocarrefour, 83(4), 295-305.

GENDRON P-J, 2016, Analyse de la diversité de l'élevage à partir des données du RA sur la base de la typologie Inosys dans les deux territoires d'approfondissements : la Communauté de communes du Bocage Bourbonnais Communauté de communes du Pays de Saint-Flour Margeride, rapport DRAAF Auvergne-Rhône-Alpes, 18p.

HERAULT-FOURNIER C., MERLE A. PRIGENT-SIMONIN A-H, 2012, Comment les consommateurs perçoivent-ils la proximité à l'égard d'un circuit court alimentaire ? Management et Avenir, $\mathrm{N}^{\circ} 53,16-33$.

FELDMANN C., HAMM U., 2015, Consumers' perceptions and preferences for local fodd: A review Food Quality and Preference, 40, 152-164.

HIRCZAK M., MOLLARD A., 2004, Qualité des produits et qualité de l'environnement : des espaces convergents ? L'exemple de la région Rhône-Alpes, Revue d'Economie Régionale et Urbaine, 5, 845 - 868.

HIRCZAK M., 2011, L'interrelation complexe entre signes de qualité et environnement en France, Norois, 219, 75-88.

Institut de l'Elevage et de la CNE, 2017, Chiffres clés 2016. http://www.idele.fr

LANCASTER K., 1991, Modern Consumer Theory, Edward Elgar Publishing Limited, 242 p.

MARGETIC C., 1999, Filières agro-alimentaires «de qualité » et territoires : l'exemple des filières viande dans le Nord-Pas-de-Calais, Sud-Ouest Européen, 6, 61-68.

Ministère de l'Agriculture et de l'Alimentation, 2018, Agreste Synthèses - Consommation avril $2018-\mathrm{n}^{\circ} 2018 / 322$

MOALLA M., MOLLARD A., PECQUEUR B., 2005, Offre de produits-services territorialisés et approche lancastérienne de la demande de biens combinés in Torre A., Filippi M. "Proximités et changements socio-économiques dans les mondes ruraux", Editions de l'INRA, collection Science en marche.

MOLLARD A., 2001, Qualité et développement territorial : une grille d'analyse théorique à partir de la rente, Économie rurale, 263, 16-34.

MOLLARD A., PECQUEUR B., MOALLA M., 2005, Offre de produits et services territorialisés et approche lancastérienne de la demande de biens combinés, in Proximités et changements socio-économiques dans les mondes ruraux, Torre A., Filippi M. (éds.), Versailles: INRA Editions, Collection "Le Point Sur", 73-93.

MOLLARD A., RAMBONILAZA M., VOLLET D., 2006, Aménités environnementales et rente territoriale sur un marché de services différenciés: le marché des gîtes ruraux labellisés en France, Revue d'Économie Politique, 2 (03-04), 251-276.

PECQUEUR B., 2001, Qualité et développement territorial : l'hypothèse du panier de biens et de services territorialisés, 2001, Économie Rurale, janvier-février, 161, 37-49.

RICHARD F., CHEVALLIER M., DELLIER J., LAGARDE V., 2014, Circuits courts agroalimentaires de proximité en Limousin: performance économique et processus de gentrification rurale, Norois, 230, 21-39.

ROUX E., VOLLET D., PECQUEUR B., 2007, Coordination d'acteurs et valorisation des ressources territoriales, les cas de l'Aubrac et des Baronnies, Économie Rurale, 293, 20-36. 
RYSCHAWY J., TICHIT M., BERTRAND S., ALLAIRE G., PLANTUREUX S., AZNAR O., PERROT C., GUINOT C., JOSIEN E., LASSEUR J., AUBERT C., TCHAKERIAN E., DISENHAUS C., 2015, Comment évaluer les services rendus par l'élevage? Une approche méthodologique sur le cas de la France, INRA Prod. Anim., 28, 23-37.

TARDIEU S., RUDELLE A., 1962, Abattage et dépeçage du porc, In: CNRS, l'Aubrac, étude ethnologique, linguistique, agronomique et économique d'un établissement humain, Editions du CNRS, Paris, Tome 6.2, Ethnologie contemporaine, 155-184. 